\title{
Weak laws of large numbers for weighted sums of Banach space valued fuzzy random variables
}

\section{Yun Kyong Kim}

Department of Information \& Communication Engineering, Dongshin University, Geonjaero 253, Naju, Jeonnam, 520-714, Korea

\begin{abstract}
In this paper, we present some results on weak laws of large numbers for weighted sums of fuzzy random variables taking values in the space of normal and upper-semicontinuous fuzzy sets with compact support in a separable real Banach space. First, we give weak laws of large numbers for weighted sums of strong-compactly uniformly integrable fuzzy random variables. Then, we consider the case that the weighted averages of expectations of fuzzy random variables converge. Finally, weak laws of large numbers for weighted sums of strongly tight or identically distributed fuzzy random variables are obtained as corollaries.
\end{abstract}

Keywords: Fuzzy sets, Random sets, Fuzzy random variables, Weak law of large numbers, Compactly uniform integrability, Tightness, Weighted sum.

\section{Introduction}

In recent years, the theory of fuzzy sets introduced by Zadeh [1] has been extensively studied and applied the fields of statistics and probability. Statistical inference for fuzzy probability models led to the requirement for laws of large numbers to ensure consistency in estimation problems.

Since Puri and Ralescu [2] introduced the concept of fuzzy random variables as a natural generalization of random sets, several authors have studied laws of large numbers for fuzzy random variables. Among others, several variants of strong law of large numbers (SLLN) for independent fuzzy random variables were built on the basis of SLLN for independent random

(c) This is an Open Access article distributed under the terms of the Creative Commons Attribution Non-Commercial License (http://creativecommons.org/licenses/ by-nc/3.0// which permits unrestricted noncommercial use, distribution, and reproduction in any medium, provided the original work is properly cited. sets. A rich variety of SLLN for fuzzy random variables can be found in the literature, e.g., Colub et al. [3,4], Feng [5], Fu and Zhang [6], Inoue [7], Klement et al. [8], Li and Ogura [9], Molchanov [10], Proske and Puri [11].

However, weak laws of large numbers (WLLN) for fuzzy random variables are not as popular as SLLN. Taylor et al. [12] obtained WLLN for fuzzy random variables in a separable Banach space under varying hypotheses of independence, exchangeability, and tightness. Joo [13] established WLLN for convex-compactly uniformly integrable fuzzy random variables taking values in the space of fuzzy numbers in a finite-dimensional Euclidean space.

Generalizing the above results for sums of fuzzy random variables to the case of weighted sums is a significant problem. In this regard, Guan and $\mathrm{Li}$ [14] obtained some results on WLLN for weighted sums of fuzzy random variables under a restrictive condition, and Joo et 
al. [15] established some results on strong convergence for weighted sums of fuzzy random variables different from those of Guan and Li [14]. Moreover, Kim [16] studied WLLN for weighted sums of level-continuous fuzzy random variables.

The purpose of this paper is to present some results on WLLN for the weighted sum of fuzzy random variables taking values in the space of normal and upper-semicontinuous fuzzy sets with compact support in a real separable Banach space. First, we give WLLN for the weighted sum of strong-compactly uniformly integrable fuzzy random variables. Then, we give WLLN for the weighted sum of fuzzy random variables such that the weighted averages of its expectations are convergent.

\section{Preliminaries}

Let $Y$ be a real separable Banach space with norm $|\cdot|$ and let $\mathbf{K}(Y)$ denote the family of all non-empty compact subsets of $Y$. Then the space $\mathbf{K}(Y)$ is metrizable by the Hausdorff metric $h$ defined by

$$
h(A, B)=\max \left\{\operatorname{supinf}_{a \in A}|a-b|, \sup _{b \in B} \inf _{a \in A}|a-b|\right\} .
$$

A norm of $A \in \mathbf{K}(Y)$ is defined by

$$
\|A\|=h(A,\{0\})=\sup _{a \in A}|a| .
$$

It is well-known that $\mathbf{K}(Y)$ is complete and separable with respect to the Hausdorff metric $h$ (See Debreu [17]).

The addition and scalar multiplication on $\mathbf{K}(Y)$ are defined as usual:

$$
A \oplus B=\{a+b: a \in A, b \in B\}, \lambda A=\{\lambda a: a \in A\}
$$

for $A, B \in \mathbf{K}(Y)$ and $\lambda \in R$.

The convex hull and closed convex hull of $A \subset Y$ are denoted by $\operatorname{co}(A)$ and $\overline{c o}(A)$, respectively. If $\operatorname{dim}(Y)<\infty$ and $A \in \mathbf{K}(Y)$, then $\operatorname{co}(A) \in \mathbf{K}(Y)$. But if $\operatorname{dim}(Y)=\infty$, it is well-known that $c o(A)$ may not be an element of $\mathbf{K}(Y)$ even though $A \in \mathbf{K}(Y)$, but $\overline{c o}(A) \in \mathbf{K}(Y)$ if $A \in \mathbf{K}(Y)$.

Let $\mathbf{F}(Y)$ denote the family of all fuzzy sets $u: Y \rightarrow[0,1]$ with the following properties;

(i) $u$ is normal, i.e., there exists $x \in Y$ such that $u(x)=1$;

(ii) $u$ is upper-semicontinuous;

(iii) $\operatorname{supp} u=\operatorname{cl}\{x \in Y: u(x)>0\}$ is compact, where $\operatorname{cl}(A)$ denotes the closure of $A$ in $Y$.
For a fuzzy subset $u$ of $Y$, the $\alpha$-level set of $u$ is defined by

$$
L_{\alpha} u=\left\{\begin{array}{lcc}
\{x: u(x) \geq \alpha\} & \text { if } & 0<\alpha \leq 1, \\
\operatorname{supp} u & \text { if } & \alpha=0 .
\end{array}\right.
$$

Then it follows immediately that $u \in \mathbf{F}(Y)$ if and only if $L_{\alpha} u \in \mathbf{K}(Y)$ for each $\alpha \in[0,1]$. If we denote $c l\{x \in Y: u(x)>$ $\alpha$ ) by $L_{\alpha^{+}} u$, then

$$
\lim _{\beta \downarrow \alpha} h\left(L_{\beta} u, L_{\alpha^{+}} u\right)=0 .
$$

The linear structure on $\mathbf{F}(Y)$ is also defined as usual;

$$
\begin{aligned}
(u \oplus v)(z) & =\sup _{x+y=z} \min (u(x), v(y)), \\
(\lambda u)(z) & = \begin{cases}u(z / \lambda), & \text { if } \lambda \neq 0, \\
\tilde{0}(z), & \text { if } \lambda=0,\end{cases}
\end{aligned}
$$

for $u, v \in \mathbf{F}(Y)$ and $\lambda \in R$, where $\tilde{0}=I_{\{0\}}$ denotes the indicator function of $\{0\}$.

Then it is known that for each $\alpha \in[0,1], L_{\alpha}(u \oplus v)=L_{\alpha} u \oplus$ $L_{\alpha} v$ and $L_{\alpha}(\lambda u)=\lambda L_{\alpha} u$.

Recall that a fuzzy subset $u$ of $Y$ is said to be convex if

$u(\lambda x+(1-\lambda) y) \geq \min (u(x), u(y))$ for $x, y \in Y$ and $\lambda \in[0,1]$.

The convex hull of $u$ is defined by

$$
\operatorname{co}(u)=\inf \{v: v \text { is convex and } v \geq u\} .
$$

Then it is known that for each $\alpha \in[0,1], L_{\alpha} \operatorname{co}(u)=\operatorname{co}\left(L_{\alpha} u\right)$.

If $Y$ is finite dimensional space and $u \in \mathbf{F}(Y)$, then $c o(u) \in$ $\mathbf{F}(Y)$. But if $Y$ is infinite dimensional space, it may not be true. So we need the notion of the closed convex hull of $u$. The closed convex hull $\overline{c o}(u)$ of $u$ is defined by

$$
\overline{c o}(u)=\inf \{v \in \mathbf{F}(Y): v \text { is convex and } v \geq u\} .
$$

Then it is well-known that $\overline{c o}(u) \in \mathbf{F}(Y), L_{\alpha} \overline{c o}(u)=\overline{c o}\left(L_{\alpha} u\right)$ for each $\alpha \in[0,1]$ and

$$
\overline{c o}(u \oplus v)=\overline{c o}(u) \oplus \overline{c o}(v), \overline{c o}(\lambda u)=\lambda \overline{c o}(u) .
$$

The uniform metric $d_{\infty}$ and norm $\|\cdot\|$ on $\mathbf{F}(Y)$ as usual;

$$
\begin{gathered}
d_{\infty}(u, v)=\sup _{0 \leq \alpha \leq 1} h\left(L_{\alpha} u, L_{\alpha} v\right), \\
\|u\|=d_{\infty}(u, \tilde{0})=\left\|L_{0} u\right\|=\sup _{x \in L_{0} u}|x| .
\end{gathered}
$$


It is well-known that $\left(\mathbf{F}(Y), d_{\infty}\right)$ is complete but is not separable (see Klement et al. [8]).

\section{Main Results}

Throughout this paper, let $(\Omega, \mathcal{A}, P)$ be a probability space. A set-valued function $X: \Omega \rightarrow(\mathbf{K}(Y), h)$ is called a random set if it is measurable. A random set $X$ is said to be integrably bounded if $E\|X\|<\infty$. The expectation of integrably bounded random set $X$ is defined by

$$
E(X)=\{E(\xi): \xi \in L(\Omega, Y) \text { and } \xi(\omega) \in X(\omega) \text { a.s. }\}
$$

where $L(\Omega, Y)$ denotes the class of all $Y$-valued random variables $\xi$ such that $E|\xi|<\infty$.

A fuzzy set valued function $\tilde{X}: \Omega \rightarrow \mathbf{F}(Y)$ is called a fuzzy random variable (or fuzzy random set) if for each $\alpha \in[0,1], L_{\alpha} \tilde{X}$ is a random set. It is well-known that if $\tilde{X}: \Omega \rightarrow\left(\mathbf{F}(Y), d_{\infty}\right)$ is measurable, then $\tilde{X}$ is a fuzzy random variable. But the converse is not true (For details, see Colubi et al. [18], Kim [19]).

A fuzzy random set $\tilde{X}$ is said to be integrably bounded if $E\|\tilde{X}\|<\infty$. The expectation of integrably bounded fuzzy random variable $\tilde{X}$ is a fuzzy subset $E(\tilde{X})$ of $Y$ defined by

$$
E(\tilde{X})(x)=\sup \left\{\alpha \in[0,1]: x \in E\left(L_{\alpha} \tilde{X}\right)\right\}
$$

For more details for expectations of random sets and fuzzy random variables, the readers may refer to Li et al. [20].

Let $\left\{\tilde{X}_{n}\right\}$ be a sequence of integrably bounded fuzzy random variables and $\left\{\lambda_{n i}\right\}$ be a double array of real numbers that not necessarily Toeplitz but satisfying

$$
\sum_{i=1}^{\infty}\left|\lambda_{n i}\right| \leq C \text { for each } n
$$

where $C>0$ is a constant not depending on $n$.

The problem that we will consider is to establish sufficient conditions for

$d_{\infty}\left(\oplus_{i=1}^{n} \lambda_{n i} \tilde{X}_{i}, \oplus_{i=1}^{n} \lambda_{n i} \overline{c o}\left(E \tilde{X}_{i}\right)\right) \rightarrow 0$ in probability as $n \rightarrow \infty$, where $\overline{c o}\left(E \tilde{X}_{i}\right)$ denotes the closed convex hull of $E\left(\tilde{X}_{i}\right)$.

To this end, we need the concepts of tightness and compact uniform integrability for a sequence of fuzzy random variables.

Definition 3.1. Let $\left\{X_{n}\right\}$ be a sequence of random sets.

(i) $\left\{X_{n}\right\}$ is said to be tight if for each $\varepsilon>0$, there exists a compact subset $\mathcal{K}$ of $(\mathbf{K}(Y), h)$ such that

$$
P\left(X_{n} \notin \mathcal{K}\right)<\varepsilon \text { for all } n
$$

(ii) $\left\{X_{n}\right\}$ is said to be compactly uniformly integrable(CUI) if for each $\varepsilon>0$, there exists a compact subset $\mathcal{K}$ of $(\mathbf{K}(Y), h)$ such that

$$
\int_{\left\{X_{n} \notin \mathcal{K}\right\}}\left\|X_{n}\right\| d P<\varepsilon \text { for all } n .
$$

Definition 3.2. Let $\left\{\tilde{X}_{n}\right\}$ be a sequence of fuzzy random variables.

(i) $\left\{\tilde{X}_{n}\right\}$ is said to be level-wise independent if for each $\alpha \in[0,1]$, the sequence $\left\{L_{\alpha} \tilde{X}_{n}\right\}$ of random sets is independent.

(ii) $\left\{\tilde{X}_{n}\right\}$ is said to be independent if the sequence $\left\{\sigma\left(\tilde{X}_{n}\right)\right\}$ of $\sigma$-fields is independent, where $\sigma(\tilde{X})$ is the smallest $\sigma$-field which $L_{\alpha} \tilde{X}$ is measurable for all $\alpha \in[0,1]$.

(iii) $\left\{\tilde{X}_{n}\right\}$ is said to be tight if for each $\varepsilon>0$, there exists a compact subset $\mathcal{K}$ of $(\mathbf{K}(Y), h)$ such that

$$
P\left(L_{\alpha} \tilde{X}_{n} \notin \mathcal{K}\right)<\varepsilon \text { for all } n \text { and all } \alpha \in[0,1] .
$$

(iv) $\left\{\tilde{X}_{n}\right\}$ is said to be strongly tight if for each $\varepsilon>0$, there exists a compact subset $K$ of $\left(\mathbf{F}(Y), d_{\infty}\right)$ such that

$$
P\left(\tilde{X}_{n} \notin K\right)<\varepsilon \text { for all } n \text {. }
$$

(v) $\left\{\tilde{X}_{n}\right\}$ is said to be compactly uniformly integrable (CUI) if for each $\varepsilon>0$ there exists a compact subset $\mathcal{K}$ of $(\mathbf{K}(Y), h)$ such that

$$
\int_{\left\{L_{\alpha} \tilde{X}_{n} \notin \mathcal{K}\right\}}\left\|L_{\alpha} \tilde{X}_{n}\right\| d P<\varepsilon \text { for all } n \text { and all } \alpha \in[0,1] .
$$

(vi) $\left\{\tilde{X}_{n}\right\}$ is said to be strong-compactly uniformly integrable (SCUI) if for each $\varepsilon>0$ there exists a compact subset $K$ of $\left(\mathbf{F}(Y), d_{\infty}\right)$ such that

$$
\int_{\left\{\tilde{X}_{n} \notin K\right\}}\left\|\tilde{X}_{n}\right\| d P<\varepsilon \text { for all } n .
$$

It is trivial that strong-compactly uniform integrability (resp. strong tightness) implies compactly uniform integrability (resp. 
tightness). But, the converse is not true even though $Y$ is finite dimensional.

First, we establish weak law of large numbers for weighted sums of strong-compactly uniformly integrable fuzzy random variables.

Theorem 3.3. Let $\left\{\tilde{X}_{n}\right\}$ be a sequence of integrably bounded fuzzy random variables and let $\left\{\lambda_{n i}\right\}$ be a double array of real numbers satisfying

$$
\sum_{i=1}^{\infty}\left|\lambda_{n i}\right| \leq C \text { for each } n
$$

Then

$$
\begin{aligned}
d_{\infty}\left(\oplus_{i=1}^{n} \lambda_{n i} \tilde{X}_{i}, \oplus_{i=1}^{n} \lambda_{n i} \overline{c o}\left(E \tilde{X}_{i}\right)\right) & \rightarrow 0 \\
& \text { in probability as } n \rightarrow \infty
\end{aligned}
$$

if and only if for each $\alpha \in[0,1]$,

$$
\begin{aligned}
h\left(\oplus_{i=1}^{n} \lambda_{n i} L_{\alpha} \tilde{X}_{i}, \oplus_{i=1}^{n} \lambda_{n i} \overline{c o}\left(E L_{\alpha} \tilde{X}_{i}\right)\right) & \rightarrow 0 \\
& \text { in probability as } n \rightarrow \infty .
\end{aligned}
$$

To prove the above theorem, we need some lemmas obtained by Kim (submitted) which is based on the characterization of relatively compact subsets of $\left(\mathbf{F}(Y), d_{\infty}\right)$ established by Greco and Moschen [21]. For easy references, we list them without proof.

Lemma 3.4. Let $K$ be a relatively compact subset of $\left(\mathbf{F}(Y), d_{\infty}\right)$. Then $\{\overline{c o}(u): u \in K\}$ is also relatively compact in $\left(\mathbf{F}(Y), d_{\infty}\right)$.

Recall that we can define the concept of convexity on $\mathbf{F}(Y)$ as in the case of a vector space even though $\mathbf{F}(Y)$ is not a vector space. That is, $K \subset \mathbf{F}(Y)$ is said to be convex if $\lambda u \oplus(1-\lambda) v \in$ $K$ whenever $u, v \in K$ and $0 \leq \lambda \leq 1$. Also, the convex hull $\operatorname{co}(K)$ of $K$ is defined to be the intersection of all convex sets that contains $K$. Then we can easily show that $c o(K)$ is equal to the family of consisting of all fuzzy sets in the form $\lambda_{1} u_{1} \oplus$ $\cdots \oplus \lambda_{k} u_{k}$, where $u_{1}, \ldots, u_{k}$ are any elements of $K, \lambda_{1}, \ldots, \lambda_{k}$ are nonnegative real numbers satisfying $\sum_{i=1}^{k} \lambda_{i}=1$ and $k=$ $2,3, \ldots$.

Lemma 3.5. Let $K$ be a relatively compact subset of $\left(\mathbf{F}(Y), d_{\infty}\right)$. Then $\operatorname{co}(K)$ is also relatively compact in $\left(\mathbf{F}(Y), d_{\infty}\right)$.

For a fixed partition $\pi: 0=\alpha_{0}<\alpha_{1}<\cdots<\alpha_{r}=1$ of $[0,1]$, we define

$g_{\pi}: \mathbf{F}(Y) \rightarrow \mathbf{F}(Y), \quad g_{\pi}(u)(x)=\sum_{k=1}^{r} \alpha_{k-1} I_{A_{k-1} \backslash A_{k}}(x)+I_{A_{r}}(x)$, where $A_{k}=L_{\alpha_{k}} u$.

Then it follows that

$$
L_{\alpha} g_{\pi}(u)= \begin{cases}L_{\alpha_{1}} u, & \text { if } \quad 0 \leq \alpha \leq \alpha_{1} \\ L_{\alpha_{k}} u, & \text { if } \alpha_{k-1}<\alpha \leq \alpha_{k}, k=2, \ldots, r\end{cases}
$$

From this fact, we can prove easily that

$$
g_{\pi}(u \oplus v)=g_{\pi}(u) \oplus g_{\pi}(v) \text { and } g_{\pi}(\lambda u)=\lambda g_{\pi}(u)
$$

Lemma 3.6. Let $K$ be a relatively compact subset of $\left(\mathbf{F}(Y), d_{\infty}\right)$. Then for each natural number $m$, there exists a partition $\pi_{m}$ of $[0,1]$ such that

$$
\sup _{u \in K} d_{\infty}\left(u, g_{\pi_{m}}(u)\right)<\frac{1}{m}
$$

We are now in a position to prove the main theorem.

Proof of Theorem 3. The necessity is trivial. To prove the sufficiency, We can assume that $C=1$ without loss of generality. Let $\varepsilon>0$ and $0<\delta<1$ be given. By strong-compactly uniform integrability of $\left\{\tilde{X}_{n}\right\}$, we can choose a compact subset $K$ of $\left(\mathbf{F}(Y), d_{\infty}\right)$ such that

$$
\int_{\left\{\tilde{X}_{n} \notin K\right\}}\left\|\tilde{X}_{n}\right\| d P<\varepsilon \delta / 12 \text { for all } n \text {. }
$$

Without loss of generality, we may assume that $\tilde{0} \in K, K$ is convex and symmetric (i.e., $(-1) u \in K$ if $u \in K$ ), and that $K$ contains $\overline{c o}(u)$ for all $u \in K$ by lemmas 4 and 5 .

By lemma 6 , we choose a partition $\pi_{m}: 0=\alpha_{m, 0}<\alpha_{m, 1}<$ $\cdots<\alpha_{m, r_{m}}$ of $[0,1]$ such that

$$
\sup _{u \in K} d_{\infty}\left(u, g_{\pi_{m}}(u)\right)<\frac{1}{m}<\varepsilon / 6 .
$$

Now we denote

$$
\tilde{U}_{n}=I_{\left\{\tilde{X}_{n} \in K\right\}} \tilde{X}_{n}, \tilde{V}_{n}=I_{\left\{\tilde{X}_{n} \notin K\right\}} \tilde{X}_{n} .
$$

Then by assumptions of $K$ and $\lambda_{n i}$, we have

$$
\overline{c o}\left(E \tilde{U}_{i}\right) \in K \text { and } \oplus_{i=1}^{n} \lambda_{n i} \overline{c o}\left(E \tilde{U}_{i}\right) \in K
$$

Thus by (2),

$$
d_{\infty}\left(\oplus_{i=1}^{n} \lambda_{n i} \overline{c o}\left(E \tilde{U}_{i}\right), g_{\pi_{m}}\left[\oplus_{i=1}^{n} \lambda_{n i} \overline{c o}\left(E \tilde{U}_{i}\right)\right]\right)<\varepsilon / 6
$$


Then we have

$$
\begin{aligned}
& d_{\infty}\left(\oplus_{i=1}^{n} \lambda_{n i} \overline{c o}\left(E \tilde{X}_{i}\right), \oplus_{i=1}^{n} \lambda_{n i} g_{\pi_{m}}\left[\overline{c o}\left(E \tilde{X}_{i}\right)\right]\right) \\
\leq \quad & d_{\infty}\left(\oplus_{i=1}^{n} \lambda_{n i} \overline{c o}\left(E \tilde{U}_{i}\right), \oplus_{i=1}^{n} \lambda_{n i} g_{\pi_{m}}\left[\overline{c o}\left(E \tilde{U}_{i}\right)\right]\right) \\
& \quad+d_{\infty}\left(\oplus_{i=1}^{n} \lambda_{n i} \overline{c o}\left(E \tilde{V}_{i}\right), \oplus_{i=1}^{n} \lambda_{n i} g_{\pi_{m}}\left[\overline{c o}\left(E \tilde{V}_{i}\right)\right]\right) \\
\leq \quad & \left\|\oplus_{i=1}^{n} \lambda_{n i} \overline{c o}\left(E \tilde{V}_{i}\right)\right\|+\left\|g_{\pi_{m}}\left(\oplus_{i=1}^{n} \lambda_{n i} \overline{c o}\left(E \tilde{V}_{i}\right)\right)\right\| \\
& \quad+\varepsilon / 6 \quad \text { by }(3) \\
\leq \quad & 2 \sum_{i=1}^{n}\left|\lambda_{n i}\right|\left\|\overline{c o}\left(E \tilde{V}_{i}\right)\right\|+\varepsilon / 6 \\
\leq \quad & \varepsilon \delta / 6+\varepsilon / 6<\varepsilon / 3 \quad \text { by }(1) .
\end{aligned}
$$

Hence we obtain

$$
\begin{aligned}
d_{\infty} & \left(\oplus_{i=1}^{n} \lambda_{n i} \tilde{X}_{i}, \oplus_{i=1}^{n} \lambda_{n i} \overline{c o}\left(E \tilde{X}_{i}\right)\right) \\
\leq \quad d_{\infty} & \left(\oplus_{i=1}^{n} \lambda_{n i} \tilde{X}_{i}, \oplus_{i=1}^{n} \lambda_{n i} g_{\pi_{m}}\left(\tilde{X}_{i}\right)\right) \\
& +d_{\infty}\left(\oplus_{i=1}^{n} \lambda_{n i} g_{\pi_{m}}\left(\tilde{X}_{i}\right), \oplus_{i=1}^{n} \lambda_{n i} g_{\pi_{m}}\left[\overline{c o}\left(E \tilde{X}_{i}\right)\right]\right)+\varepsilon / 3
\end{aligned}
$$

This implies that

$$
\begin{aligned}
& P\left\{d_{\infty}\left(\oplus_{i=1}^{n} \lambda_{n i} \tilde{X}_{i}, \oplus_{i=1}^{n} \lambda_{n i} \overline{c o}\left(E \tilde{X}_{i}\right)\right)>\varepsilon\right\} \\
\leq \quad & P\left\{d_{\infty}\left(\oplus_{i=1}^{n} \lambda_{n i} \tilde{X}_{i}, \oplus_{i=1}^{n} \lambda_{n i} g_{\pi_{m}}\left(\tilde{X}_{i}\right)\right)>\varepsilon / 3\right\} \\
& +P\left\{d_{\infty}\left(\oplus_{i=1}^{n} \lambda_{n i} g_{\pi_{m}}\left(\tilde{X}_{i}\right), \oplus_{i=1}^{n} \lambda_{n i} g_{\pi_{m}}\left[\overline{c o}\left(E \tilde{X}_{i}\right)\right]\right)>\varepsilon / 3\right\} \\
= & (\mathrm{I})+(\mathrm{II}) .
\end{aligned}
$$

For (I), we first note that

$$
\begin{aligned}
& d_{\infty}\left(\oplus_{i=1}^{n} \lambda_{n i} \tilde{X}_{i}, \oplus_{i=1}^{n} \lambda_{n i} g_{\pi_{m}}\left(\tilde{X}_{i}\right)\right) \\
\leq \quad & d_{\infty}\left(\oplus_{i=1}^{n} \lambda_{n i} \tilde{U}_{i}, \oplus_{i=1}^{n} \lambda_{n i} g_{\pi_{m}}\left(\tilde{U}_{i}\right)\right) \\
& +d_{\infty}\left(\oplus_{i=1}^{n} \lambda_{n i} \tilde{V}_{i}, \oplus_{i=1}^{n} \lambda_{n i} g_{\pi_{m}}\left(\tilde{V}_{i}\right)\right) \\
\leq & \left.\left\|\oplus_{i=1}^{n} \lambda_{n i} \tilde{V}_{i}\right\|+\| \oplus_{i=1}^{n} \lambda_{n i} g_{\pi_{m}}\left(\tilde{V}_{i}\right)\right) \|+\varepsilon / 6 \text { by }(2) \\
\leq & 2\left\|\oplus_{i=1}^{n} \lambda_{n i} \tilde{V}_{i}\right\|+\varepsilon / 6 .
\end{aligned}
$$

And so

$$
\begin{aligned}
\text { (I) } & \leq P\left\{\left\|\oplus_{i=1}^{n} \lambda_{n i} \tilde{V}_{i}\right\|>\varepsilon / 12\right\} \leq \frac{12}{\varepsilon} E\left\|\oplus_{i=1}^{n} \lambda_{n i} \tilde{V}_{i}\right\| \\
& \leq \frac{12}{\varepsilon} \sum_{i=1}^{n}\left|\lambda_{n i}\right| E\left\|\tilde{V}_{i}\right\| \leq \frac{12}{\varepsilon} \frac{\varepsilon \delta}{12}=\delta \quad \text { by (1) }
\end{aligned}
$$

Now for (II), since

$$
\begin{gathered}
d_{\infty}\left(\oplus_{i=1}^{n} \lambda_{n i} g_{\pi_{m}}\left(\tilde{X}_{i}\right), \oplus_{i=1}^{n} \lambda_{n i} g_{\pi_{m}}\left[\overline{c o}\left(E \tilde{X}_{i}\right)\right]\right) \\
=\max _{1 \leq k \leq r_{m}} h\left(\oplus_{i=1}^{n} \lambda_{n i} L_{\alpha_{m, k}} \tilde{X}_{i}, \oplus_{i=1}^{n} \lambda_{n i} L_{\alpha_{m, k}} \overline{c o} E\left(\tilde{X}_{i}\right)\right)
\end{gathered}
$$

we have

$$
\begin{aligned}
\text { (II) } & \leq \sum_{k=1}^{r_{m}} P\left\{h\left(\oplus_{i=1}^{n} \lambda_{n i} L_{\alpha_{m, k}} \tilde{X}_{i}, \oplus_{i=1}^{n} \lambda_{n i} L_{\alpha_{m, k}} \overline{c o} E\left(\tilde{X}_{i}\right)\right)>\frac{\varepsilon}{3} r_{m}\right\} \\
& \leq \delta
\end{aligned}
$$

for sufficiently large $n$ by our assumption. This completes the proof.

Corollary 3.7. Let $\left\{X_{n}\right\}$ be a sequence of strongly tight fuzzy random variables such that

$$
\sup _{n} E\left\|\tilde{X}_{n}\right\|^{p}=M<\infty \text { for some } p>1 .
$$

Then

$$
\begin{aligned}
& d_{\infty}\left(\oplus_{i=1}^{n} \lambda_{n i} \tilde{X}_{i}, \oplus_{i=1}^{n} \lambda_{n i} \overline{c o}\left(E \tilde{X}_{i}\right)\right) \rightarrow 0 \\
& \text { in probability as } n \rightarrow \infty
\end{aligned}
$$

if and only if for each $\alpha \in[0,1]$,

$$
h\left(\oplus_{i=1}^{n} \lambda_{n i} L_{\alpha} \tilde{X}_{i}, \oplus_{i=1}^{n} \lambda_{n i} \overline{c o}\left(E L_{\alpha} \tilde{X}_{i}\right)\right) \rightarrow 0
$$

in probability as $n \rightarrow \infty$.

By applying Theorem 3, we can obtain WLLN for level-wise independent case.

Theorem 3.8. Let $\left\{\tilde{X}_{n}\right\}$ be a sequence of level-wise independent and strong-compactly uniformly integrable fuzzy random variables. Then for any Toeplitz sequence $\left\{\lambda_{n i}\right\}$ satisfying $\max _{1 \leq i \leq n}\left|\lambda_{n i}\right|=O\left(n^{-\gamma}\right)$ for some $\gamma>0$,

$$
\begin{aligned}
d_{\infty}\left(\oplus_{i=1}^{n} \lambda_{n i} \tilde{X}_{i}, \oplus_{i=1}^{n} \lambda_{n i} \overline{c o} E\left(\tilde{X}_{i}\right)\right) & \rightarrow 0 \\
& \text { in probability as } n \rightarrow \infty .
\end{aligned}
$$

Proof. Let $\varepsilon>0$ and $0<\delta<1$ be given and $K$ be a compact subset of $\left(\mathbf{F}(Y), d_{\infty}\right)$ such that

$$
\begin{array}{r}
\sup _{u \in K}\|u\|<\infty, \\
E\left(I_{\left\{\tilde{X}_{n} \notin K\right\}}\left\|\tilde{X}_{n}\right\|\right)<\varepsilon \delta / 4 C .
\end{array}
$$

Let us denote

$$
\tilde{U}_{n}=I_{\left\{\tilde{X}_{n} \in K\right\}} \tilde{X}_{n} \text { and } \tilde{V}_{n}=I_{\left\{\tilde{X}_{n} \notin K\right\}} \tilde{X}_{n} .
$$

Then since

$$
\begin{aligned}
& d_{\infty}\left(\oplus_{i=1}^{n} \lambda_{n i} \tilde{X}_{i}, \oplus_{i=1}^{n} \lambda_{n i} \overline{c o}\left(E \tilde{X}_{i}\right)\right) \leq \tilde{U}_{\infty}\left(\oplus_{i=1}^{n} \lambda_{n i} \tilde{U}_{i}, \oplus_{i=1}^{n} \lambda_{n i} \overline{c o}\left(E \tilde{U}_{i}\right)\right) \\
& \quad+d_{\infty}\left(\oplus_{i=1}^{n} \lambda_{n i} \tilde{V}_{i}, \oplus_{i=1}^{n} \lambda_{n i} \overline{c o}\left(E \tilde{V}_{i}\right)\right),
\end{aligned}
$$


we have that

$$
\begin{aligned}
& P\left\{d_{\infty}\left(\oplus_{i=1}^{n} \lambda_{n i} \tilde{X}_{i}, \oplus_{i=1}^{n} \lambda_{n i} \overline{c o}\left(E \tilde{X}_{i}\right)\right)>\varepsilon\right\} \\
& \leq P\left\{d_{\infty}\left(\oplus_{i=1}^{n} \lambda_{n i} \tilde{U}_{i}, \oplus_{i=1}^{n} \lambda_{n i} \overline{c o}\left(E \tilde{U}_{i}\right)>\varepsilon / 2\right\}\right. \\
& +P\left\{d_{\infty}\left(\oplus_{i=1}^{n} \lambda_{n i} \tilde{V}_{i}, \oplus_{i=1}^{n} \lambda_{n i} \overline{c o}\left(E \tilde{V}_{i}\right)\right)>\varepsilon / 2\right\} \\
& =(\mathrm{I})+(\mathrm{II}) \text {. }
\end{aligned}
$$

For (I), we note that for each $\alpha \in[0,1]$, the sequence $\left\{L_{\alpha} \tilde{U}_{n}\right\}$ of random sets is independent and tight. Since (4) implies

$$
\sup _{n} E\left\|\tilde{U}_{n}\right\|^{r}<\infty \text { for all } r>1
$$

we have that by Corollary 3.2 of Taylor and Inoue [22],

$$
h\left(\oplus_{i=1}^{n} \lambda_{n i} L_{\alpha} \tilde{U}_{i}, \oplus_{i=1}^{n} \lambda_{n i} \overline{c o}\left(E L_{\alpha} \tilde{U}_{i}\right)\right) \rightarrow 0 \text { a.s. as } n \rightarrow \infty
$$

By Corollary 7, this implies that (I) $\rightarrow 0$ as $n \rightarrow \infty$.

Now for (II), since

$$
\begin{aligned}
E\left[d_{\infty}\left(\oplus_{i=1}^{n} \lambda_{n i} \tilde{V}_{i}, \oplus_{i=1}^{n} \lambda_{n i} \overline{c o}\left(E \tilde{V}_{i}\right)\right)\right] \\
\leq E\left[\sum_{i=1}^{n}\left|\lambda_{n i}\right|\left\|\tilde{V}_{i}||+\sum_{i=1}^{n}\left|\lambda_{n i}\right| E\right\| \tilde{V}_{i}||\right] \\
\quad=2 \sum_{i=1}^{n}\left|\lambda_{n i}\right| E \| \tilde{V}_{i}||<\varepsilon \delta / 2 \text { by }(5)
\end{aligned}
$$

we have that

$$
\text { (II) } \leq \frac{2}{\varepsilon} E\left[d_{\infty}\left(\oplus_{i=1}^{n} \lambda_{n i} \tilde{V}_{i}, \oplus_{i=1}^{n} \lambda_{n i} \overline{c o}\left(E \tilde{V}_{i}\right)\right)\right]<\delta
$$

Thus for large $n$,

$$
P\left\{d_{\infty}\left(\oplus_{i=1}^{n} \lambda_{n i} \tilde{X}_{i}, \oplus_{i=1}^{n} \lambda_{n i} \overline{c o}\left(E \tilde{X}_{i}\right)\right)>\varepsilon\right\}<\delta
$$

which completes the proof.

Corollary 3.9. Let $\left\{\tilde{X}_{n}\right\}$ be a sequence of level-wise independent and strongly tight fuzzy random variables such that

$$
\sup _{n} E\left\|\tilde{X}_{n}\right\|^{p}=M<\infty \text { for some } p>1 \text {. }
$$

Then for any Toeplitz sequence $\left\{\lambda_{n i}\right\}$ satisfying $\max _{1 \leq i \leq n}\left|\lambda_{n i}\right|=$ $O\left(n^{-\gamma}\right)$ for some $\gamma>0$,

$$
\begin{aligned}
d_{\infty}\left(\oplus_{i=1}^{n} \lambda_{n i} \tilde{X}_{i}, \oplus_{i=1}^{n} \lambda_{n i} \overline{c o}\left(E \tilde{X}_{i}\right)\right) & =0 \\
& \text { in probability as } n \rightarrow \infty .
\end{aligned}
$$

Unfortunately, the following example shows that a sequence of identically distributed fuzzy random variables may not be strong-compactly uniformly integrable.

Example. Let $Y=R$. For $0<\lambda<1$, we define

$$
u_{\lambda}(x)= \begin{cases}1, & \text { if } x=0 \\ \lambda, & \text { if } 0<|x| \leq 1 \\ 0, & \text { elsewhere }\end{cases}
$$

Then

$$
L_{\alpha} u_{\lambda}= \begin{cases}\{0\}, & \text { if } \lambda<\alpha \leq 1 \\ \{x:|x| \leq 1\}, & \text { if } 0 \leq \alpha \leq \lambda\end{cases}
$$

and so $d_{\infty}\left(u_{\lambda}, u_{\delta}\right)=1$ for $\lambda \neq \delta$.

Now we let $\Omega=(0,1), \mathcal{A}=$ the Lebesque $\sigma$-field and $P$ be the Lebesgue measure. and let $\left\{\tilde{X}_{n}\right\}$ be a sequence of identically distributed fuzzy random variables with $\tilde{X}$ defined by

$$
\tilde{X}: \Omega \rightarrow \mathbf{F}(R), \tilde{X}(\lambda)=\tilde{u}_{\lambda}
$$

Suppose that $0<\varepsilon<1$ and that there is a compact subset $K$ of $\left(\mathbf{F}(R), d_{\infty}\right)$ such that

$$
P(\tilde{X} \notin K)=\int_{\left\{\tilde{X}_{n} \notin K\right\}}\left\|\tilde{X}_{n}\right\|<\varepsilon .
$$

Then $K$ necessarily contains a set of the form

$$
K_{J}=\left\{u_{\lambda}: \lambda \in J\right\},
$$

where $P(J)>1-\varepsilon$. But this is impossible because $K_{J}$ contains a sequence $\left\{u_{\lambda_{n}}: \lambda_{n} \in J\right\}$ which does not have any convergent subsequence.

The above example implies that Theorem 3 cannot be applied for identically distributed fuzzy random variables. Guan and Li [14] gave an WLLN for weighted sums of level-wise independent fuzzy random variables under the assumption that $\left\{\oplus_{i=1}^{n} \lambda_{n i} \overline{c o}\left(E \tilde{X}_{i}\right)\right\}$ is convergent. The next theorem is slightly different from the result of Guan and Li [14].

Theorem 3.10. Let $\left\{\tilde{X}_{n}\right\}$ be a sequence of integrably bounded fuzzy random variables such that for some $v \in \mathbf{F}(Y)$,

$$
\lim _{n \rightarrow 0} d_{\infty}\left(\oplus_{i=1}^{n} \lambda_{n i} \overline{c o}\left(E \tilde{X}_{i}\right), v\right)=0 .
$$

Then

$$
d_{\infty}\left(\oplus_{i=1}^{n} \lambda_{n i} \tilde{X}_{i}, \oplus_{i=1}^{n} \lambda_{n i} \operatorname{coE}\left(\tilde{X}_{i}\right)\right) \rightarrow 0
$$
in probability as $n \rightarrow \infty$ 
if and only if for each $\alpha \in[0,1]$,

$$
h\left(\oplus_{i=1}^{n} \lambda_{n i} L_{\alpha} \tilde{X}_{i}, \oplus_{i=1}^{n} \lambda_{n i} \overline{c o}\left(E L_{\alpha} \tilde{X}_{i}\right)\right) \rightarrow 0
$$

in probability as $n \rightarrow \infty$

and

$h\left(\oplus_{i=1}^{n} \lambda_{n i} L_{\alpha^{+}} \tilde{X}_{i}, \oplus_{i=1}^{n} \lambda_{n i} \overline{c o}\left(E L_{\alpha^{+}} \tilde{X}_{i}\right)\right) \rightarrow 0$

in probability as $n \rightarrow \infty$.

Proof. To prove the sufficiency, it suffices to prove that

$$
d_{\infty}\left(\oplus_{i=1}^{n} \lambda_{n i} \tilde{X}_{i}, v\right) \rightarrow 0 \text { in probability as } n \rightarrow \infty .
$$

Let $\tilde{S}_{n}=\oplus_{i=1}^{n} \lambda_{n i} \tilde{X}_{i}$ and let $\varepsilon>0$ be given. By Lemma 4 of Guan and Li [8], there exists a partition $0=\alpha_{0}<\alpha_{1}<\cdots<$ $\alpha_{r}=1$ such that

$$
h\left(L_{\alpha_{k}} v, L_{\alpha_{k-1}^{+}} v\right)<\varepsilon / 6 \text { for all } k=1, \ldots, r \text {. }
$$

Then by our assumption, we can find a natural number $N$ such that

$h\left(\overline{c o}\left(E L_{\alpha} \tilde{S}_{i}\right), L_{\alpha} v\right)<\varepsilon / 6$ for all $\alpha \in[0,1]$ and $n \geq N$.

First we note that if $A_{1} \subset A \subset A_{2}$ and $B_{1} \subset B \subset B_{2}$, then

$$
h(A, B) \leq \max \left[h\left(A_{1}, B_{2}\right), h\left(A_{2}, B_{1}\right)\right] .
$$

If $0<\alpha \leq 1$, then $\alpha_{k-1}<\alpha \leq \alpha_{k}$ for some $k$. Since $L_{\alpha_{k}} \tilde{S}_{n} \subset$ $L_{\alpha} \tilde{S}_{n} \subset L_{\alpha_{k-1}^{+}} \tilde{S}_{n}$ and $L_{\alpha_{k}} v \subset L_{\alpha} v \subset L_{\alpha_{k-1}^{+}} v$, we have that for $n \geq N$,

$$
\begin{array}{r}
h\left(L_{\alpha} \tilde{S}_{n}, L_{\alpha} v\right) \\
\leq \max \left[h\left(L_{\alpha_{k}} \tilde{S}_{n}, L_{\alpha_{k-1}^{+}} v\right), h\left(L_{\alpha_{k-1}^{+}} \tilde{S}_{n}, L_{\alpha_{k}} v\right)\right] \\
\leq \max \left[h\left(L_{\alpha_{k}} \tilde{S}_{n}, L_{\alpha_{k}} v\right), h\left(L_{\alpha_{k-1}^{+}} \tilde{S}_{n}, L_{\alpha_{k-1}^{+}} v\right)\right]+\varepsilon / 6 \\
\leq \max \left[h \left(L_{\alpha_{k}} \tilde{S}_{n}, \overline{c o}\left(E L_{\alpha_{k}} \tilde{S}_{n}\right), h\left(L_{\alpha_{k-1}^{+}} \tilde{S}_{n}, \overline{c o}\left(E L_{\alpha_{k-1}^{+}} \tilde{S}_{n}\right)\right]\right.\right. \\
+\varepsilon / 3 \quad \text { by (7). }
\end{array}
$$

Thus for $n \geq N$,

$$
\begin{aligned}
d_{\infty}\left(\tilde{S}_{n}, v\right)= & \max _{1 \leq k \leq r} \sup _{\alpha_{k-1}<\alpha \leq \alpha_{k}} h\left(L_{\alpha} \tilde{S}_{n}, L_{\alpha} v\right) \\
\leq & \max _{1 \leq k \leq r} h\left(L_{\alpha_{k}} \tilde{S}_{n}, \overline{c o}\left(E L_{\alpha_{k}} \tilde{S}_{n}\right)\right) \\
& +\max _{1 \leq k \leq r} h\left(L_{\alpha_{k-1}^{+}} \tilde{S}_{n}, \overline{c o}\left(E L_{\alpha_{k-1}^{+}} \tilde{S}_{n}\right)\right)+\varepsilon / 3 .
\end{aligned}
$$

Therefore, by assumption we obtain

$$
\begin{aligned}
& P\left\{d_{\infty}\left(\tilde{S}_{n}, v\right)>\varepsilon\right\} \\
& \leq P\left\{\max _{1 \leq k \leq r} h\left(L_{\alpha_{k}} \tilde{S}_{n}, \overline{c o}\left(E L_{\alpha_{k}} \tilde{S}_{n}\right)\right)>\varepsilon / 3\right\} \\
& \quad+P\left\{\max _{1 \leq k \leq r} h\left(L_{\alpha_{k-1}^{+}} \tilde{S}_{n}, \overline{c o}\left(E L_{\alpha_{k-1}^{+}} \tilde{S}_{n}\right)\right)>\varepsilon / 3\right\} \\
& \leq \sum_{k=1}^{r} P\left\{h\left(L_{\alpha_{k}} \tilde{S}_{n}, \overline{c o}\left(E L_{\alpha_{k}} \tilde{S}_{n}\right)\right)>\varepsilon / 3\right\} \\
& \quad+\sum_{k=1}^{r} P\left\{h\left(L_{\alpha_{k-1}^{+}} \tilde{S}_{n}, \overline{c o}\left(E L_{\alpha_{k-1}^{+}} \tilde{S}_{n}\right)\right)>\varepsilon / 3\right\} \\
& \rightarrow \quad 0 \text { as } n \rightarrow \infty .
\end{aligned}
$$

This completes the proof.

Corollary 3.11. Let $\left\{\tilde{X}_{n}\right\}$ be a sequence of identically distributed fuzzy random variables with $E\left\|\tilde{X}_{1}\right\|<\infty$, and $\left\{\lambda_{n i}\right\}$ be a double sequence of real numbers satisfying

$$
\lim _{n \rightarrow \infty} \sum_{i=1}^{n} \lambda_{n i}=\lambda \text { for some } \lambda \in R .
$$

Then

$$
d_{\infty}\left(\oplus_{i=1}^{n} \lambda_{n i} \tilde{X}_{i}, \lambda \overline{c o}\left(E \tilde{X}_{1}\right)\right) \rightarrow 0
$$

in probability as $n \rightarrow \infty$

if and only if for each $\alpha \in[0,1]$

$$
\begin{aligned}
h\left(\oplus_{i=1}^{n} \lambda_{n i} L_{\alpha} X_{i}, \overline{c o}\left(E L_{\alpha} X_{1}\right)\right) & \rightarrow 0 \\
& \text { in probability as } n \rightarrow \infty
\end{aligned}
$$

and

$$
\begin{aligned}
h\left(\oplus_{i=1}^{n} \lambda_{n i} L_{\alpha^{+}} X_{i}, \overline{c o}\left(E L_{\alpha^{+}} X_{1}\right)\right) & \rightarrow 0 \\
& \text { in probability as } n \rightarrow \infty .
\end{aligned}
$$

Proof. The necessity is trivial. To prove the sufficiency, we note that

$$
\begin{aligned}
& d_{\infty}\left(\oplus_{i=1}^{n} \lambda_{n i} \overline{c o}\left(E \tilde{X}_{i}\right), \lambda \overline{c o}\left(E \tilde{X}_{1}\right)\right) \\
& \quad \leq\left|\sum_{i=1}^{n} \lambda_{n i}-\lambda\right|\left\|\overline{c o}\left(E \tilde{X}_{1}\right)\right\| \rightarrow 0 \text { as } n \rightarrow \infty .
\end{aligned}
$$

Since

$$
\begin{aligned}
d_{\infty}\left(\oplus_{i=1}^{n} \lambda_{n i} \tilde{X}_{i}, \lambda \overline{c o}\left(E \tilde{X}_{1}\right)\right) \\
\leq d_{\infty}\left(\oplus_{i=1}^{n} \lambda_{n i} \tilde{X}_{i}, \oplus_{i=1}^{n} \lambda_{n i} \overline{\overline{c o}}\left(E \tilde{X}_{i}\right)\right) \\
\quad+d_{\infty}\left(\oplus_{i=1}^{n} \lambda_{n i} \overline{c o}\left(E \tilde{X}_{i}\right), \lambda \overline{c o}\left(E \tilde{X}_{1}\right)\right)
\end{aligned}
$$

the desired result follows immediately. 


\section{Conclusions}

In this paper, we obtained two types of necessary and sufficient conditions under which weak laws of large numbers for weighted sums of fuzzy random variables hold. One is the case of strong-compactly uniformly integrable fuzzy random variables. The other is the case that the weighted averages of its expectations converge. The former includes a strongly tight case and the latter contains the identically distributed case. We also provided WLLN for weighted sums of level-wise independent and strong-compactly uniformly integrable (or strongly tight) fuzzy random variables.

It remains an open problem whether we can obtain a generalization for the above WLLN to the case of compactly uniform integrability.

\section{References}

[1] L.A. Zadeh, "Fuzzy sets," Information and Control, vol. 8, no. 3 pp. 338-353, Jun. 1965. http://dx.doi.org/10.1016/ S0019-9958(65)90241-X

[2] M. L. Puri and D. A. Ralescu, "Fuzzy random variables," Journal of Mathematical Analysis and Applications, vol. 114, no. 2, pp. 409-422, Mar. 1986. http://dx.doi.org/10. 1016/0022-247X(86)90093-4

[3] A. Colubi, M. Lopez-Diiaz, J. S. Domiinguez-Menchero, and M. A. Gil, "A generalized strong law of large numbers," Probability Theory and Related Fields, vol. 114, no. 3, pp. 401-417, Jun. 1999. http://dx.doi.org/0.1007/ s004400050229

[4] A. Colubi, J. S. Dominguez-Menchero, M. Lopez-Diaz, and R. Korner, "A method to derive strong laws of large numbers for random upper semicontinuous functions," Statistics \& Probability Letters, vol. 53, no. 3, pp. 269275, Jun. 2001. http://dx.doi.org/10.1016/S0167-7152(01) 00057-8

[5] Y. Feng, "An approach to generalize laws of large numbers for fuzzy random variables," Fuzzy Sets and Systems, vol. 128, no. 2, pp. 237-245, Jun. 2002. http://dx.doi.org/10. 1016/S0165-0114(01)00142-7

[6] K. A. Fu and L. X. Zhang, "Strong laws of large numbers for arrays of rowwise independent random compact sets and fuzzy random sets," Fuzzy Sets and Systems, vol. 159, no. 24, pp. 3360-3368, Dec. 2008. http://dx.doi.org/10. 1016/j.fss.2008.06.012

[7] H. Inoue, "A strong law of large numbers for fuzzy random sets," Fuzzy Sets and Systems, vol. 41, no. 3, pp. 285291, Jun. 1991. http://dx.doi.org/10.1016/0165-0114(91) 90132-A

[8] E. P. Klement, M. L. Puri, and D. A. Ralescu, "Limit theorems for fuzzy random variables," Proceedings of the Royal Society A, vol. 407, no. 1832, pp. 171-182, Sep. 1986. http://dx.doi.org/10.1098/rspa.1986.0091

[9] S. Li and Y. Ogura, "Strong laws of large numbers for independent fuzzy set-valued random variables," Fuzzy Sets and Systems, vol. 157, no. 19, pp. 2569-2578, Oct. 2006. http://dx.doi.org/10.1016/j.fss.2003.06.011

[10] I. S. Molchanov, "On strong laws of large numbers for random upper semicontinuous functions," Journal of Mathematical Analysis and Applications, vol. 235, no. 1, pp. 349355, Jul. 1999. http://dx.doi.org/10.1006/jmaa.1999.6403

[11] F. N. Proske and M. L. Puri, "Strong law of large numbers for banach space valued fuzzy random variables," Journal of Theoretical Probability, vol. 15, no. 2, pp. 543-551, Apr. 2002. http://dx.doi.org/10.1023/A:1014823228848

[12] R. L. Taylor, L. Seymour, and Y. Chen, "Weak laws of large numbers for fuzzy random sets," Nonlinear Analysis: Theory, Methods \& Applications, vol. 47, no. 2, pp. 1245-1256, Aug. 2001. http://dx.doi.org/10.1016/ S0362-546X(01)00262-0

[13] S. Y. Joo, "Weak laws of large numbers for fuzzy random variables," Fuzzy Sets and Systems, vol. 147, no. 3, pp. 453-464, Nov. 2004. http://dx.doi.org/10.1016/j.fss.2004. 02.005

[14] L. Guan and S. Li, "Laws of large numbers for weighted sums of fuzzy set-valued random variables," International Journal of Uncertainty, Fuzziness and Knowledge-Based Systems, vol. 12, no. 6, pp. 811-825, Dec. 2004. http: //dx.doi.org/10.1142/S0218488504003223

[15] S. Y. Joo, Y. K. Kim, and J. S. Kwon, "Strong convergence for weighted sums of fuzzy random sets," Information Sciences, vol. 176, no. 8, pp. 1086-1099, Apr. 2006. http: //dx.doi.org/10.1016/j.ins.2005.02.002 
[16] Y. K. Kim, "Weak convergence for weighted sums of levelcontinuous fuzzy random variables," Journal of Fuzzy Logic and Intelligent Systems, vol. 14, no. 7, pp. 852-856, Dec. 2004.

[17] G Debreu, "Integration of correspondences," in Proceedings of the 5th Berkeley Symposium on Mathematical Statistics and Probability, Berkeley, CA, 1967, pp. 351372 .

[18] A. Colubi, J. S. Domıinguez-Menchero, M. Lopez-Diaz, and D. A. Ralescu, "On the formalization of fuzzy random variables," Information Sciences, vol. 133, no. 1-2, pp. 36, Mar. 2001. http://dx.doi.org/10.1016/S0020-0255(01) 00073-1

[19] Y. K. Kim, "Measurability for fuzzy valued functions," Fuzzy Sets and Systems, vol. 129, no. 1, pp. 105-109, Jul. 2002. http://dx.doi.org/10.1016/S0165-0114(01)00121-X

[20] S. Li, Y. Ogura, and V. Kreinovich, Limit Theorems and Applications of Set-Valued and Fuzzy Set-Valued Random Variables, Boston: Kluwer Academic Publishers, 2002.

[21] G. H. Greco and M. P. Moschen, "Supremum metric and relatively compact sets of fuzzy sets," Nonlinear Analysis:
Theory, Methods \& Applications, vol. 64, no. 6, pp. 13251335, Mar. 2006. http://dx.doi.org/10.1016/j.na.2005.06. 038

[22] R. L. Taylor and H. Inoue, "Convergence of weighted sums of random sets," Stochastic Analysis and Applications, vol. 3, no. 3, pp. 379-396, 1985. http://dx.doi.org/ $10.1080 / 07362998508809069$

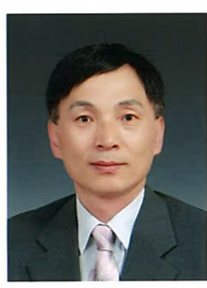

Yun Kyong Kim received the B.S degree in Mathematics Education from Kangwon National University in 1978. And then, he received the M.S. and Ph.D degrees in Mathematics from Korea University in 1983 and 1988, respectively. From 1989 to 2002, he was a professor in Dept. of Mathematics, Dongshin University. Since 2002, he has been a professor in Dept. of Information \& Communication Engineering, Dongshin University. His research interests center on Fuzzy Probability, Fuzzy Analysis and Related Fields.

E-mail: ykkim@dsu.ac.kr 